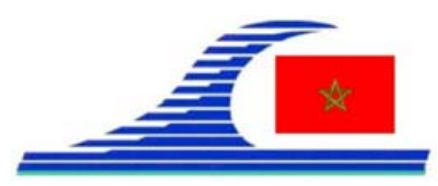

Conférence Méditerranéenne Côtière et Maritime

EDITION 2, TANGER, MAROC (2011)

Coastal and Maritime Mediterranean Conference

Disponible en ligne - http://www.paralia.fr - Available online

\title{
Etat de la contamination des côtes atlantiques de Nouadhibou par les métaux lourds (Mauritanie)
}

\section{Mohammed MHAMADA ${ }^{\mathbf{1}}$, Mohammed OULD MOHAMED CHEIHK ${ }^{\mathbf{1}}$, Aly DARDIGE ${ }^{2}$, Hassan ER-RAIOUI ${ }^{1}$}

\author{
1. Université Abdelmalek Essâadi, Ancienne route de l'aviation, BP. 416, Tanger, \\ Maroc.h.erraioui@fstt.ac.ma \\ 2. Office d'Inspection Sanitaire des Produits de la Pêche et de l'Aquaculture (ONISPA), \\ Nouadibou, Mauritanie.
}

\section{Résumé :}

Le littoral atlantique de Nouadhibou, qui abrite une importante faune sous-marine et compte des sites de pêche parmi les plus productifs, est le siège de rejets urbains et des activités portuaires importantes. L'examen de l'état de la contamination à travers l'analyse des métaux lourds dans des bivalves et des produits halieutiques montre des concentrations élevées en Cd notamment chez les praires (3.696 à $11.543 \mu \mathrm{g} \mathrm{g}^{-1}$ ) et les sardinelles $\left(600 \mu \mathrm{g} \mathrm{g}^{-1}\right)$. Ces résultats reflètent l'impact des rejets urbains et des activités portuaires au niveau de la baie du Lévrier, caractérisée par un hydrodynamisme faible et une évaporation intense et au niveau de la zone économique exclusive de la Mauritanie (ZEE) soumise à un hydroclimat qui entraine un risque élevé de dispersion de polluants. Mots-clés :

Littoral atlantique de Nouadhibou - Zone économique exclusive - Produits de pêche Métaux lourds - Baie du Lévrier

\section{Introduction}

Les eaux marines sur les côtes et particulièrement au contact des concentrations industrielles et des ports montrent les formes de dégradation les plus inquiétantes. Parmi les substances soupçonnées, figurent les métaux lourds. Ne faisant pas l'objet de biodégradation, ils peuvent s'accumuler dans les chaînes alimentaires pour atteindre des seuils toxiques (CUMONT, 1984). Le suivi de la contamination par les métaux, au moyen des bivalves et des produits halieutiques, est de pratique courante. Ils sont utilisés comme indicateurs de pollution car doués de pouvoir de bioaccumulation.

Les côtes mauritaniennes s'étendent sur près de $720 \mathrm{~km}$. La zone économique exclusive de la Mauritanie (ZEE) couvre près de $230000 \mathrm{~km}^{2}$ dont $9000 \mathrm{~km}^{2}$ occupés, par l'ensemble Baie du Lévrier-banc d'Arguin qui constitue des sites écologiques à potentiel de ressources importantes et diversifiées. Les côtes sont continuellement soumises à une forte pression des activités humaines et reçoivent des rejets industriels et urbains en plus des activités portuaires et notamment du port minéralier. Situé sur les frontières nord du littoral mauritanien à proximité de Nouadhibou qui la longe sur plus de $15 \mathrm{~km}$, la baie du 
La connaissance de la Mer :

un vecteur du développement durable en Méditerranée

Lévrier se trouve confrontée à plusieurs sources de pollution correspondant aux rejets urbains et aux installations industrielles et portuaires, en plus d'une pêche intense. La surveillance de la mer est particulièrement des zones côtières se veut une obligation pour sauvegarder le potentiel aquacole des eaux marines et ses ressources naturelles. Cette étude, réalisée au niveau de la Baie du Lévrier et sur quelques espèces de poisson prélevées dans la ZEE sur une période d'un an, constitue une contribution pour mettre l'accent sur l'état de salubrité du littoral (figure 1). Trois métaux ( $\mathrm{Pb}, \mathrm{Cd}$ et $\mathrm{Hg}$ ) ont été dosés dans la chaire des moules, des praires, des poulpes et des sardinelles.

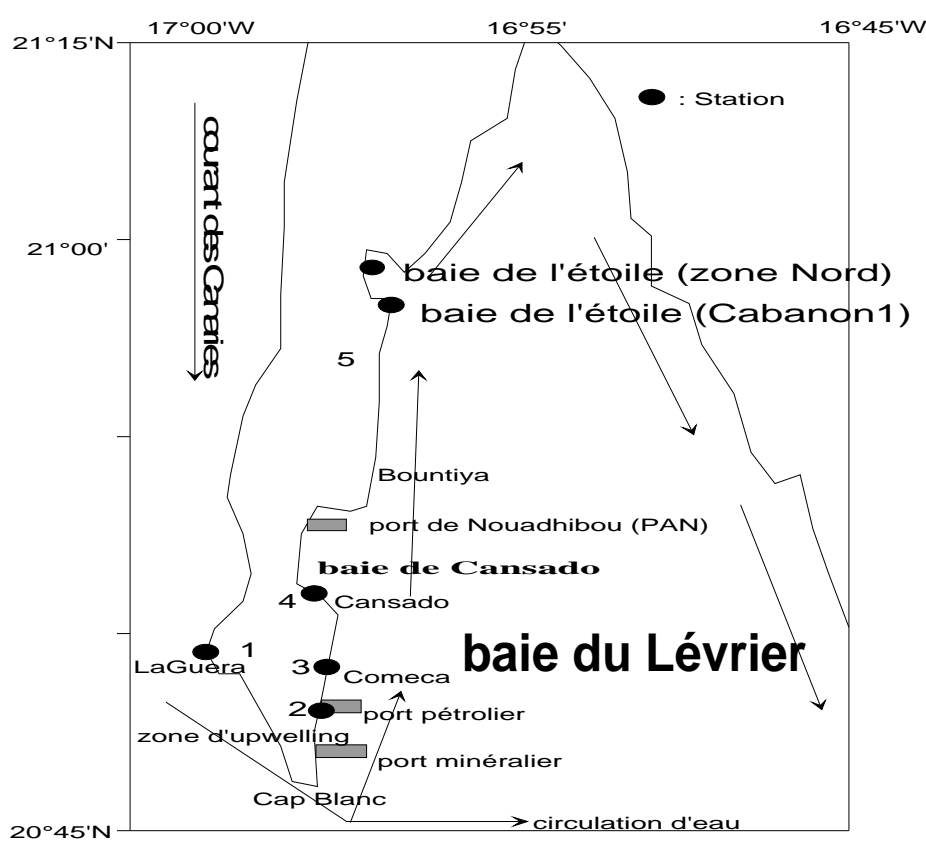

Figure 1. Zone maritime rosalina (stations de prélèvements des échantillons de moules).

\section{Matériels et méthodes}

Les moules ont été détachés à l'aide d'un couteau en inox. Les praires ont été collectés à l'aide d'une drague montée à bord du bateau de recherche à une profondeur de $-6 \mathrm{~m}$ et $-10 \mathrm{~m}$ et à 500 et $800 \mathrm{~m}$ de la côte. Les poulpes et les sardinelles ont été péchés dans la ZEE. Les échantillons ont été rincés à l'eau de mer et placés dans une glacière isotherme $\left(2\right.$ et $\left.8^{\circ} \mathrm{C}\right)$ dans des sacs en polyéthylène. Dés l'arrivée au laboratoire, les échantillons sont conservés à $-20^{\circ} \mathrm{C}$. Lors de l'analyse, les échantillons sont décongelés puis décortiqués. La chaire est broyée et lyophilisée. Une fois les échantillons déshydratés, ils ont été réduit en une fine poudre homogène. La minéralisation consiste en une digestion de $0,2 \mathrm{~g}$ à $\mathrm{HNO}_{3}$. Les échantillons sont ensuite chauffés par microonde pendant 2 min 20 à $80 \%$ et 5 min à 100\%. Après minéralisation, les métaux lourds ont été analysés par spectrophotométrie d'absorption atomique Shimadzu AA 6800 équipé d'un four à graphite GFA-EX7 et piloté par un logiciel. Des solutions mères à 1000 ppm obtenues soit à partir du métal, de l'oxyde ou d'un sel ont été utilisées. Une 
série de dilution est effectuée pour obtenir une gamme de concentration appropriée, avec ppm $\left(\mathrm{mg} \mathrm{l}^{-1}\right)$ et $\mathrm{ppb}\left(\mu \mathrm{g} \mathrm{l}^{-1}\right)$.

\section{Résultats}

Les résultats montrent des concentrations élevées en $\mathrm{Cd}$. Les plus fortes teneurs sont enregistrées chez les sardinelles de la ZEE $\left(600 \mu \mathrm{g} \mathrm{g}^{-1}\right)$. Chez les praires, les teneurs en Cd fluctuent entre $3.696 \mu \mathrm{g} \mathrm{g}^{-1}$ et $11.543 \mu \mathrm{g} \mathrm{g}^{-1}$. Ceci peut être attribué à un upwelling intense qui emmènerait en surface une partie des eaux charriées par le Courant des Canaries riche en $\mathrm{Cd}$. Pour les éléments $\mathrm{Pb}$ et $\mathrm{Hg}$, les teneurs sont faibles et ne dépassent $0.338 \mu \mathrm{g} \mathrm{g}^{-1}$ et $0.013 \mu \mathrm{g} \mathrm{g}^{-1}$ respectivement.

Pour le Cd, des concentrations similaires ont été signalées par DARTIGE (2006). Dans une étude, SIDOUMOU et al. (1992) montrent que les concentrations en Cd chez des praires sont de $11,86 \mu \mathrm{g} \mathrm{g}^{-1}$ en moyenne et sont plus élevées que celles des praires de la baie de Cansado, incluse dans la baie du Lévrier et qui reçoit les déchets des ports et de la raffinerie, en plus des rejets des industries de pêche et des rejets urbains de l'agglomération de Nouadhibou. Le fonctionnement hydrologique de la ZEE, caractérise par des mouvements intenses des masses d'eaux, explique le risque de dispersion des polluants sur les côtes. Pour la baie du Lévrier, les concentrations élevées peuvent s'expliquer par la courantologie de la région. Bien que éloigné des sources de rejets, la baie correspond à un écosystème calme où les eaux se renouvellement plus lentement et où l'évaporation est intense.

Sur le littoral casablancais au Maroc, des teneurs moyennes de 1,21 de poids sec (ps) ont été enregistrées. Cependant des maximums de 3,64 $\mu \mathrm{g} \mathrm{g}^{-1}$ ps ont été signalés dans des sites sous l'influence des rejets industriels (BOUTHIR et al., 2004). En outre, CHAFIK et al. (2001) signalent des taux de 11,9 $\mathrm{gg} \mathrm{g}^{-1}$ ps dans des moules prélevées sur l'Atlantique sud du Maroc. Aussi, BANAOUI et al. (2004) ont remarqué que, malgré l'absence de source de pollution dans la zone sud du Maroc (Cape Juby au Sahara), les taux en $\mathrm{Cd}$ sont élevés $\left(6 \mu \mathrm{g} \mathrm{g}^{-1} \mathrm{ps}\right)$ et semble être attribués par à l'upwelling très intense entre le Cap Ghir et le Cap Juby.

Concernant le mercure, les travaux antérieurs mentionnent des valeurs médianes de 0,03 à $0,31 \mu \mathrm{g} \mathrm{g}^{-1}$ chez la moule Perna perna (DARTIGE, 2006). Ce résultat est confirmé par plusieurs auteurs notamment SIDOUMOU et al. (1992) qui notent une moyenne de $0,05 \mu \mathrm{g} \mathrm{g}^{-1}$ de poids sec. En outre, CUMONT et al. (1975) ont obtenu, au niveau de l'Atlantique, des valeurs de l'ordre de $0,08 \mathrm{mg} \mathrm{kg}^{-1}$ de poids sec chez des poissons. Au niveau du littoral casablancais (Maroc), les résultats affichent un maximum de $7,73 \mu \mathrm{g} \mathrm{g}^{-1}$ ps au voisinage des zones de rejets urbains, alors qu'ailleurs, les valeurs sont faibles et inférieures aux valeurs limites $\mathrm{t}\left(0,5 \mu \mathrm{g} \mathrm{g}^{-1}\right.$ de poids frais), (CEE, 2001).

Cependant, chez la moule Perna perna, OTCHERE et al. (2003) mentionnent des valeurs plus élevées au Ghana $\left(0,26 \mu \mathrm{g} \mathrm{g}^{-1} \mathrm{ps}\right)$ et au Maroc $\left(0,04\right.$ à $\left.2,6 \mu \mathrm{g} \mathrm{g}^{-1} \mathrm{ps}\right)$ (BANAOUI et al. (2004)). Pour le $\mathrm{Pb}$, des teneurs de $11,64 \mu \mathrm{g} \mathrm{g}^{-1}$ ps ont été signalées 
La connaissance de la Mer :

un vecteur du développement durable en Méditerranée

au niveau du littoral de Casa Blanca (atlantique marocain). Elles ont été attribuées aux rejets des industries dans la région (BOUTHIR et al., 2004).

\section{Conclusion}

D'un potentiel économique important, la mer constitue un levier de développement économique pour la Mauritanie. Les résultats dégagés du suivi de l'état de la salubrité du littoral, par l'analyse de certains éléments métalliques chez les organismes bivalves et les produits halieutiques (moules, praires, poulpes et sardinelles) en provenance de la zone économique exclusive et de la baie du Lévrier, montrent des concentrations faibles comparées à d'autres zones jugées polluées à l'exception du cadmium qui affiche des taux élevées à l'instar des côtes ouest africaine.

\section{Références bibliographiques}

BANAOUI A., CHIFFOLEAU J.F., MOUKRIM A., BURGEOT T., KAAYA A., AUGER D., ROZUEL E. (2004). Trace metal distribution in the mussel Perna perna along the Moroccan coast. Marine Pollution Bulletin 48, pp 378-402.

BOUTHIR F.Z., CHAFIK A., BENBRAHIM S., SOUABI S., EL MERDHY H., MESSOUDI A., SIFEDDINE M. (2004). Quality of Wilaya du grand Casablanca coastal waters (Morocco, Atlantic Ocean): the mussel Mytilus galloprovincialis as an indicator of metal contamination. Mar. Life, 14 (1-2), pp 59-70.

CHAFIK A., CHEGGOUR M., COSSA D., BENBRAHIM S., SIFEDINE M. (2001). Quality of Moroccan Atlantic coastal waters: water monitoring and mussel watching. Aquat. Living resour. 14, pp 239-249. doi:10.1016/S0990-7440(01)01123-8

CEE (2001). Reglement (CE) $N^{\circ}$ 466/2001 de la Commission du 8 mars 2001 portant fixation de teneurs maximales pour certains contaminants dans les denrées alimentaires. J.O. Commun. Europ., L 77/1, 13 p.

CUMONT G. (1984). La contamination des aliments par le mercure. Ann. Fals. Exp. Chem., 77, pp 309-320.

CUMONT G., GILlES G., BERNARD F., BRIAND M., et al. (1975). Bilan de la contamination des poissons de mer par le mercure a l'occasion d'un contrôle portant sur 3 années. Ann. Hyg. L. Fr., Méd. et Nut. 11, pp 17-25.

DARTIGE A.O.Y. (2006). Teneur en métaux lourds du mollusque Perna perna (L) prélevé dans la baie du Lévrier, Mauritanie. Thèse de l'université Libre de Bruxelles.

OTCHERE F.A., JOIRIS C.R., HOLSBEEK L. (2003). Mercury in the bivalves Anadara (Senilia) senilis, Perna perna and Crassostrea tulipa from Ghana. The Science of the Total Environment 304, pp 369-375.

SIDOUMOU Z., ROMEO M., GNASSIA-BARELLI M., NGUYEN P., CARUBA R. (1992). Détermination de la qualité des eaux du littoral mauritanien par la mesure des métaux traces chez les mollusques Donax rugosus et Venus verrucosa. Hydroécol. Appl., Tome 4, Vol 2, pp 33-41. 